\title{
High-resolution melting analysis for rapid and sensitive NOTCH1 screening in chronic lymphocytic leukemia
}

\author{
JING-JING XU ${ }^{1 *}$, FEI-RONG YAO ${ }^{2 *}$, MIN JIANG $^{3}$, YOU-TAO ZHANG $^{1}$ and FENG GUO ${ }^{1}$ \\ ${ }^{1}$ Center for Clinical Laboratory and Departments of ${ }^{2}$ Radiology, ${ }^{3}$ Blood Transfusion, \\ The First Affiliated Hospital of Soochow University, Suzhou, Jiangsu 215006, P.R. China
}

Received April 19, 2016; Accepted December 14, 2016

DOI: $10.3892 / \mathrm{ijmm} .2017 .2849$

\begin{abstract}
Chronic lymphocytic leukemia (CLL) is a biological and clinical heterogeneous disease. Activating mutations of NOTCH1 have been implicated to be associated with adverse prognosis in CLL. The objective of the present study was to develop an effective high-resolution melting (HRM) assay for detecting NOTCH1 mutations. Genomic DNA (gDNA) extracted from 133 CLL patients was screened by HRM assay, and the results were compared with the data obtained using direct sequencing. The relative sensitivity of the HRM assay and direct sequencing was evaluated using diluted gDNA with different NOTCH1 mutational frequencies. The HRM assay was able to detect and discriminate samples with NOTCHI mutations from the wild-type template in CLL. Eight of the 133 CLL patients $(6.02 \%)$ were scored positively for $\mathrm{NOTCH1}$ mutations in the HRM assay. The results of the NOTCH1 mutations detected by HRM analysis achieved $100 \%$ concordance with those determined from direct sequencing. HRM had a higher sensitivity (1\%) and shorter turn-around time (TAT), compared to direct sequencing. In conclusion, the HRM assay developed by us was confirmed to be a rapid, sensitive, and promising approach for high-throughput prognostic NOTCH1 screening in CLL. It enables real-time NOTCH1 evaluation, which is of great significance in clinical practice and may facilitate the decision-making of clinicians in CLL.
\end{abstract}

\section{Introduction}

Chronic lymphocytic leukemia (CLL) is characterized by the proliferation and accumulation of malignant $\mathrm{CD}^{+} \mathrm{CD} 19^{+}$and $\mathrm{CD}_{23}{ }^{+}$mature, monoclonal B lymphocytes in the peripheral blood, bone marrow, lymph nodes, and other secondary

Correspondence to: Professor Feng Guo, Center for Clinical Laboratory, The First Affiliated Hospital of Soochow University, 188 Shizi Street, Suzhou, Jiangsu 215006, P.R. China

E-mail: guofeng27@suda.edu.cn

*Contributed equally

Key words: NOTCH1 gene, high-resolution melting, chronic lymphocytic leukemia, direct sequencing lymphoid organs $(1,2)$. The biological behaviors and the clinical features of the disease are significantly heterogeneous $(3,4)$. Several factors, including immunoglobulin heavy chain variable region $(I G H V)$ gene mutation, cytogenetic abnormalities, zeta-chain-associated protein kinase 70 (ZAP70) and CD38 expression at diagnosis, predict prognosis and help guide therapeutic decisions (1). Recurrent gene mutations such as NOTCH1, MYD88, BIRC3 and SF3B1 confer drug resistance and adverse prognosis in CLL $(2,5,6)$.

Notch homolog 1, translocation-associated (Drosophila), also known as NOTCH1, encodes a single-pass class I transmembrane protein which exists as a non-covalently linked heterodimer (7). Functioning as a ligand-activation transcription factor, it is composed of an extracellular domain mediating ligand binding and an intracellular domain mediating signaling. Ligands, Delta-like and Jagged, following binding to the NOTCH receptor, induce proteolytic cleavage of the receptor, and result in the release and translocation of the intracellular domain to the nucleus, thus leading to transcriptional activation of multiple target genes including nuclear factor $\kappa$-light-chain-enhancer of activated $B$ cells (NF-кB) (8-10).

NOTCH1 mutations in exon 34, selectively disrupt the carboxy-terminal proline-glutamate-serine-threoninerich (PEST) domain of the protein, resulting in a truncated protein which is more stable, impaired NOTCH1 degradation, and constitutive transcriptional activation of the NOTCH1 downstream signaling including canonical and noncanonical $\mathrm{NF}-\kappa \mathrm{B}$ pathways $(5,11-18)$. Mutations in $\mathrm{NOTCH} 1$ are reported to be associated with a particularly poor outcome and may play a pivotal role in the pathogenesis and treatment resistance of CLL, and contribute to poor patient prognosis (19). Based on current research, activating mutations of $\mathrm{NOTCH} 1$ occur at a low frequency in CLL patients at diagnosis (8.3-12.6\%), but at a significantly higher frequency in patients with the more clinically aggressive $I G H V$ unmutated subtype of CLL (20.4\%), Richter syndrome (31.0\%) and chemo-refractory CLL (20.8\%) (5,13-15,20-23). Among all NOTCH1 mutations, $\sim 80 \%$ are located in exon 34 which are selected to disrupt the PEST domain.

High-resolution melting (HRM) assay is a technique for fast genotyping and high-throughput mutation of germ-line and somatic mutation analysis, which has been established since 2003 (24-26). The HRM melting profile provides a 
specific sequence-related pattern that differentiates wildtype sequences from homozygote or heterozygote variants. This method is based on real-time PCR amplification in the presence of a saturating intercalating fluorescent dye (27-29); mutations residing in the melting domain are visualized as alteration of the melting curve derived after PCR amplification by increasing the temperature and measuring the decrease of fluorescence emitted from within the double helix while the DNA strands separate $(25,30,31)$.

In the present study, we developed an accurate and sensitive HRM assay for detecting somatic NOTCH1 mutations in a total of 133 CLL patients. These gene mutations were further confirmed by direct sequencing, the gold standard. The results of HRM analysis achieved $100 \%$ concordance with those from direct sequencing. The HRM method we developed proved to be an effective, rapid and sensitive approach for $\mathrm{NOTCHI}$ screening with higher sensitivity and shorter turn-around time (TAT). It may be routinely used for the high-throughput screening of $\mathrm{NOTCH}$ in clinical CLL patients at diagnosis or at any clinical course of CLL, which is significant for decisionmaking regarding therapeutic strategies.

\section{Materials and methods}

Clinical specimens. A total of 133 CLL bone marrow samples were obtained after informed consent from patients fulfilling diagnostic and immune-phenotypic criteria for CLL at the Hematologic Department, The First Affiliated Hospital of Soochow University. Only patients at initial diagnosis without prior therapy were included in the study. Positive cut-off values were 30 and $20 \%$ for CD38 and ZAP70 expression. The mutation status of the $I G H V$ gene and cytogenetic alterations for all the patients included in this study were also analyzed. Germline $I G H V$ was defined as $\geq 98 \%$ homology. Research was performed upon approval of the Ethics Committee of The First Affiliated Hospital of Soochow University.

Genomic DNA ( $g D N A$ ) extraction. To obtain somatic DNA from CLL cells, the cells were isolated from bone marrow of CLL patients by density gradient centrifugation over lymphocyte cell separation media (Cedarlane Laboratories, Shanghai, China). After isolation, the cells were stained with anti-CD19, anti-CD5 and anti-CD20 antibodies (all from BD Biosciences, Shanghai, China), which were further analyzed by flow cytometry. Only CLL samples containing $\geq 95 \% \mathrm{CD}^{+} \mathrm{CD} 19^{+}$cells were included in the study. gDNA was extracted using gDNA isolation kits (Omega BioTek Guangzhou, Ltd., Guangzhou, China) according to the manufacturer's instructions. DNA was quantified using a NanoDrop ND-1000 fluorospectrometer (Thermo Fisher Scientific, Shanghai, China) and the A260/280 value was ensured between 1.8 and 2.0.

Cell culture. The acute lymphoblastic leukemia cell line Molt4 and the acute $\mathrm{T}$ cell leukemia cell line Jurkat, purchased from the Shanghai Institute for Biological Sciences (Shanghai, China), were cultured in RPMI-1640 medium (HyClone, Logan, UT, USA) supplemented with $10 \%$ (v/v) fetal bovine serum (FBS), $100 \mathrm{U} / \mathrm{ml}$ penicillin and $100 \mu \mathrm{g} / \mathrm{ml}$ streptomycin, $2 \mathrm{mM}$ L-glutamine and $1 \mathrm{mM}$ sodium pyruvate at $37^{\circ} \mathrm{C}$ in a humidified incubator at $5 \% \mathrm{CO}_{2}$.
HRM assay. The primers of NOTCHI used in this study are listed in Table I. The PEST domain of NOTCH1 was amplified in two fragments (Ex34a and Ex34b) with product sizes of 131 and $116 \mathrm{bp}$. The HRM assay was performed using Fast EvaGreen ${ }^{\circledR}$ qPCR Master Mix (Biotium, Hayward, CA, USA) on a LightCycler 480 instrument (Roche Diagnostics, Beijing, China). The reaction mixture in a $20 \mu \mathrm{l}$ final volume contained 1X Fast EvaGreen qPCR Master Mix, $200 \mathrm{nM}$ forward primer and $200 \mathrm{nM}$ reverse primer, $50 \mathrm{ng}$ of genomic DNA and PCR grade water. The cycling and melting conditions were as follows: $95^{\circ} \mathrm{C}$ for $2 \mathrm{~min}\left(95^{\circ} \mathrm{C}\right.$ for $5 \mathrm{sec}, 60^{\circ} \mathrm{C}$ for $35 \mathrm{sec}, 72^{\circ} \mathrm{C}$ for $25 \mathrm{sec}$ ) $\mathrm{x} 50$ cycles; HRM: $95^{\circ} \mathrm{C}$ for $1 \mathrm{~min}, 40^{\circ} \mathrm{C}$ for $1 \mathrm{~min}$, $65^{\circ} \mathrm{C}$ for $1 \mathrm{sec}$, with a continuous increase in temperature from $65^{\circ} \mathrm{C}$ to $95^{\circ} \mathrm{C}$ at the rate of $0.02^{\circ} \mathrm{C} / \mathrm{sec}$ with 25 signal acquisitions per degree; and cooling: $40^{\circ} \mathrm{C}$ for $30 \mathrm{sec}$. The melting profiles of the amplicons were analyzed using LightCycler 480 Gene-Scanning software to detect wild-type and mutations. All samples were tested in triplicate.

Direct sequencing. NOTCH1 screening (PEST domain; RefSeq NM_017617.4) in CLL was carried out by PCR amplification and direct sequencing. The primers used are listed in Table I. The PCR reaction was amplified using Platinum Taq DNA polymerase (Invitrogen, Beijing, China) and conducted under the following conditions: $94^{\circ} \mathrm{C}$ for $5 \mathrm{~min},\left(94^{\circ} \mathrm{C}\right.$ for $30 \mathrm{sec}, 60^{\circ} \mathrm{C}$ for $30 \mathrm{sec}, 72^{\circ} \mathrm{C}$ for $\left.45 \mathrm{sec}\right) \mathrm{x} 40$ cycles; $72^{\circ} \mathrm{C}$ for $10 \mathrm{~min}$. The PCR products were checked on $2 \%$ agarose gels. PCR products were purified and followed by bi-directional sequencing using an ABI 3730 DNA Analyzer (Applied Biosystems, Inc., Beijing, China). Sequencing chromatograms were analyzed using DNA Baser 3.0. Nucleotide changes detected by sequencing were all checked in Sanger's COSMIC database, and diagnosed as mutations accordingly.

Sensitivity determination. Cancer cell lines, Molt4 and Jurkat, were used. Molt4 cells harbor deletion of a CT dinucleotide in the PEST domain of the NOTCH1 gene (heterozygous for c.7541_7542delCT). Jurkat cells are wild-type of the NOTCH1 gene. Serial dilutions of the NOTCH1 mutant Molt4 cells with Jurkat cells were used to determine the sensitivity of the direct sequencing and HRM.

Statistical analysis. Data analysis was performed using GraphPad Prism 5 program (GraphPad Software, Inc., La Jolla, CA, USA). The Chi-square test was used to analyze biological features between NOTCH1-mutated and unmutated CLL groups. Differences were considered to be statistically significant when the $\mathrm{p}$-value was $<0.05$.

\section{Results}

Detection of NOTCH1 mutations by HRM analysis. For the HRM assay, we chose the optimal temperature and the primer concentration to generate specific products with efficient amplification and melting with an acceptable profile. The normalized and shifted melting curves provide the basic representation of the different genotypes, while difference plots show the difference between fluorescence of a patient's sample and a wild-type template at each temperature transition. The normalized and shifted melting curves and difference plots of 
Table I. Primers for direct sequencing or HRM assay of the PEST domain of the NOTCHI gene.

\begin{tabular}{llll}
\hline Method & Primer name & \multicolumn{1}{c}{ Sequences } & Amplicon size \\
\hline Sequencing & NOTCH1 Ex34_F & 5'-CTGGCGGTGCACACTATTCTG-3' & 327 bp \\
& NOTCH1 Ex34_R & 5'-GCGCGCCGTTTACTTGAAG-3' & \\
HRM & NOTCH1 Ex34a_F & 5'-ACAGCTACTCCTCGCCTGTG-3' & 131 bp \\
& NOTCH1 Ex34a_R & 5'-GTCGGAGACGTTGGAATGCG-3' & 116 bp \\
& NOTCH1 Ex34b_F & 5'-GTGCACACTATTCTGCCCCAG-3' & \\
\hline
\end{tabular}

HRM, high-resolution melting; PEST, proline-glutamate-serine-threonine-rich.

Table II. NOTCH1 mutations of the CLL patients.

\begin{tabular}{lccc}
\hline Mutation & $\begin{array}{c}\text { CLL } \\
\text { patients }\end{array}$ & $\begin{array}{c}\text { Direct } \\
\text { sequencing }\end{array}$ & $\begin{array}{c}\text { HRM } \\
\text { analysis }\end{array}$ \\
\hline CLL-48 & c.7541_7542delCT & p.P2514fs*4 & Mutated \\
CLL-108 & c.7541_7542delCT & p.P2514fs*4 & Mutated \\
CLL-109 & c.7541_7542delCT & p.P2514fs*4 & Mutated \\
CLL-185 & c.7541_7542delCT & p.P2514fs 4 & Mutated \\
CLL-213 & c.7541_7542delCT & p.P2514fs*4 & Mutated \\
CLL-232 & c.7535_7536insC & p.S2513fs*3 & Mutated \\
CLL-250 & c.7541_7542delCT & p.P2514fs*4 & Mutated \\
CLL-295 & c.7541_7542delCT & p.P2514fs*4 & Mutated \\
\hline
\end{tabular}

CLL, chronic lymphocytic leukemia; HRM, high-resolution melting.

$6.02 \%(8 / 133)$ of the patients were significantly different from those of the wild-type control. There were two types of shifted melting curves and difference plots in the 8 CLL samples with NOTCH1 mutations, represented by CLL-108 and CLL-232 (Fig. 1A and B). The melting curves of 7 out of 8 NOTCH1mutated patients were exactly the same. The 7 patients were confirmed to be c.7541_7542delCT (p. P2514fs*4) mutated by direct sequencing represented by CLL-108 (Fig. 1C). The melting curve of 1 out of 8 NOTCH1-mutated patients was different. This patient was confirmed to be c.7535_7536insC mutated (p.S2513fs*3) represented by CLL-232 (Fig. 1D).

Screening for NOTCH1 mutations in the PEST domain by direct sequencing. We also analyzed the NOTCH1 mutation status of 133 samples with CLL by direct sequencing and then compared the findings to that of the HRM assay. Among the 133 patients, $8(6.02 \%)$ patients, who scored positively in the HRM assay, also turned out to carry somatic mutations of the NOTCH1 gene, indicated by direct sequencing (Table II). All the mutations were heterozygous. Of them, 7 (87.5\%) cases had the 2-bp frame-shift deletion, c.7541_7542delCT (p.P2514fs*4) and 1 case had a frame-shift insertion, c.7535_7536insC (p.S2513fs"3). Another 93.98\% (125/133) of the CLL samples, showing consistency in melting curves and temp-shifted plots from those generated by the wild-type template, were implicated to be NOTCH1 wild-type in the direct sequencing. Above all, the results of the NOTCH1 mutations detected by
Table III. Characteristics of the CLL patients according to NOTCH1 mutations.

\begin{tabular}{|c|c|c|c|c|}
\hline & $\begin{array}{c}\text { All } \\
(n=133)\end{array}$ & $\begin{array}{c}\text { NOTCH1 } \\
\text { wild-type } \\
(\mathrm{n}=125)\end{array}$ & $\begin{array}{c}\text { NOTCH1 } \\
\text { mutated } \\
(\mathrm{n}=8)\end{array}$ & \\
\hline Characteristics & $\mathrm{N}(\%)$ & $\mathrm{N}(\%)$ & $\mathrm{N}(\%)$ & P-value \\
\hline$I G H V$ unmutated & $41(30.8)$ & $35(28)$ & $6(75)$ & 0.0053 \\
\hline Trisomy 12 & $30(22.6)$ & $25(20)$ & $5(62.5)$ & 0.0053 \\
\hline $\mathrm{CD} 8^{+}$expression & $21(15.8)$ & $19(15.2)$ & $2(25)$ & 0.4612 \\
\hline $\mathrm{ZAP}^{+} 0^{+}$expression & $22(16.5)$ & $20(15)$ & $2(25)$ & 0.5066 \\
\hline
\end{tabular}

CLL, chronic lymphocytic leukemia.

HRM analysis were $100 \%$ consistent with the findings from the direct sequencing.

NOTCH1 mutations are associated with adverse biological features in CLL patients. The main biological features of the CLL cohort according to NOTCH1 mutations are listed in Table III. NOTCH1-mutated CLL patients presented with higher frequencies of germline $I G H V$ unmutated $(6 / 8,75 \%)$ status and trisomy $12(5 / 8,62.5 \%)$ than these frequencies noted in the NOTCH1-unmutated patients. It is noteworthy that there were significant correlations between NOTCH1 mutations and $I G H V$ status $(\mathrm{p}=0.0053)$ or trisomy $12(\mathrm{p}=0.0053)$. There were no significant correlations between expression level of CD38 $(\mathrm{p}=0.4612)$ or ZAP70 $(\mathrm{p}=0.5066)$ with NOTCH1 mutations.

Sensitivity evaluation by HRM and direct sequencing. Cancer cell lines with known NOTCH1 genotype were used for the validation and sensitivity testing for the HRM assay and direct sequencing. The acute lymphoblastic leukemia cell line Molt4 harboring a heterozygous 7541_7542delCT (p.P2514fs*4) in NOTCHI was used as a positive control and the acute T-cell leukemia cell line Jurkat which has a wild-type NOTCH1 genotype was used as a negative control. The gDNA of the Molt4 cells was serially diluted into Jurkat gDNA at ratios of $100,40,20,10$ and $2 \%$ to yield mutant allele frequencies of 50 , $20,10,5$ and $1 \%$. The relative sensitivities of direct sequencing and HRM were evaluated using the diluted gDNA. The 
A

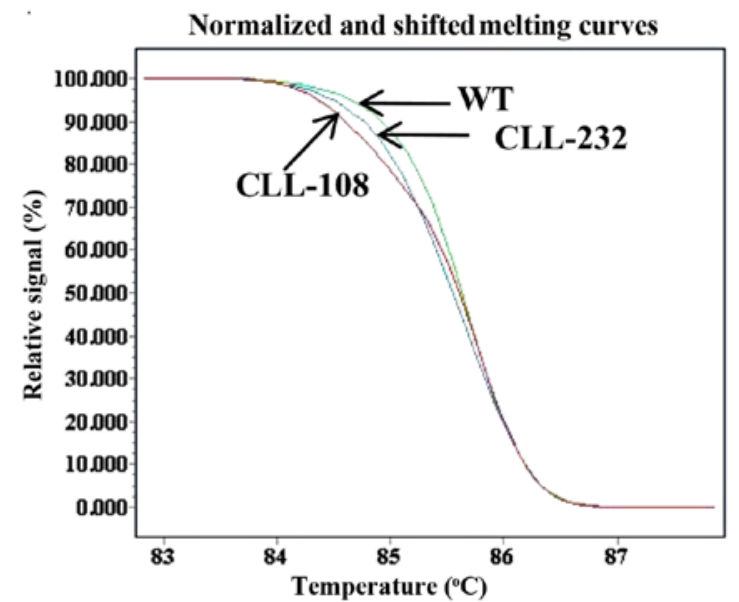

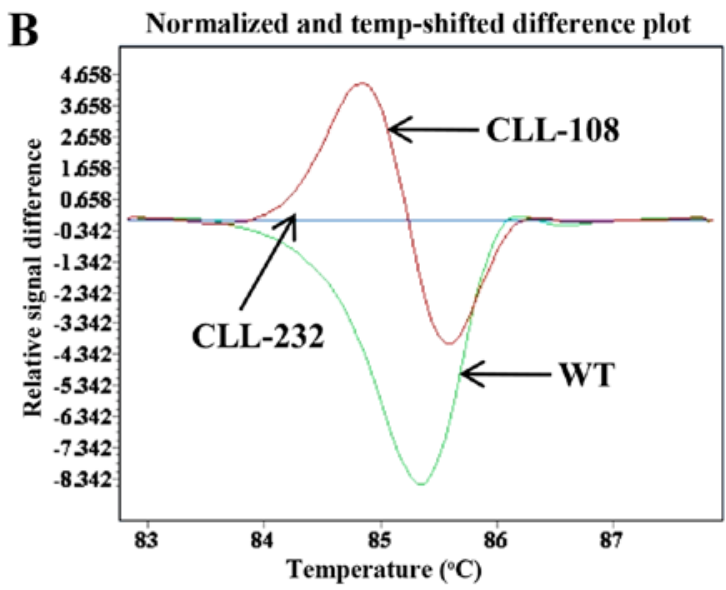

C -

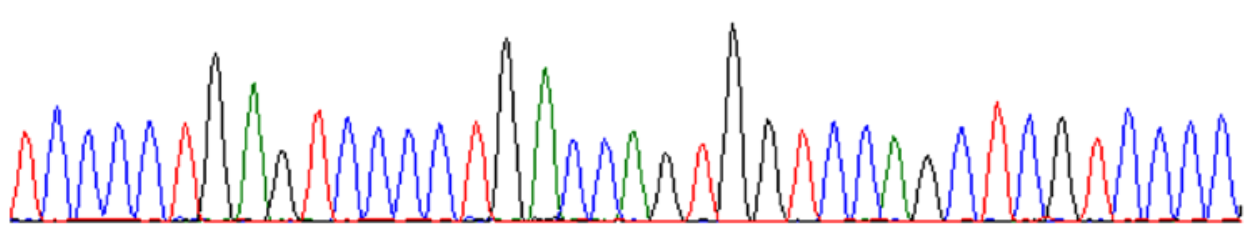

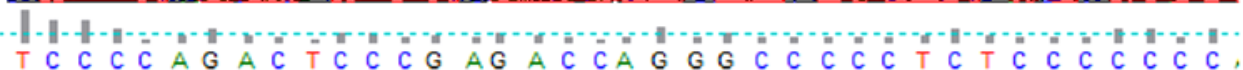

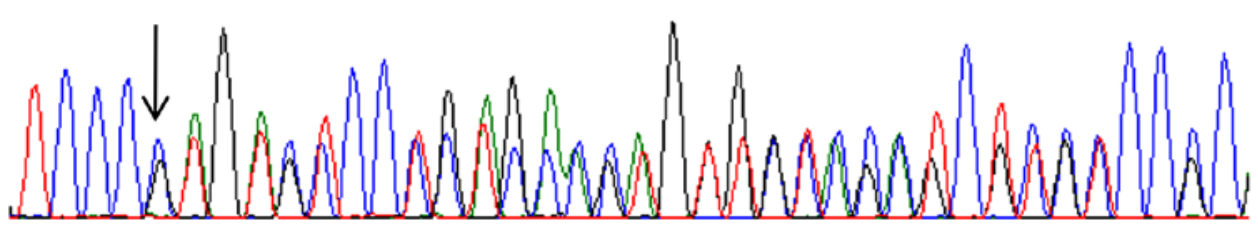

WT

CLL-108

c.7541_7542delCT

D $A C C C C G T C C C C T G A G T C C C C T G A C C A G T G G T C C A G C T C$
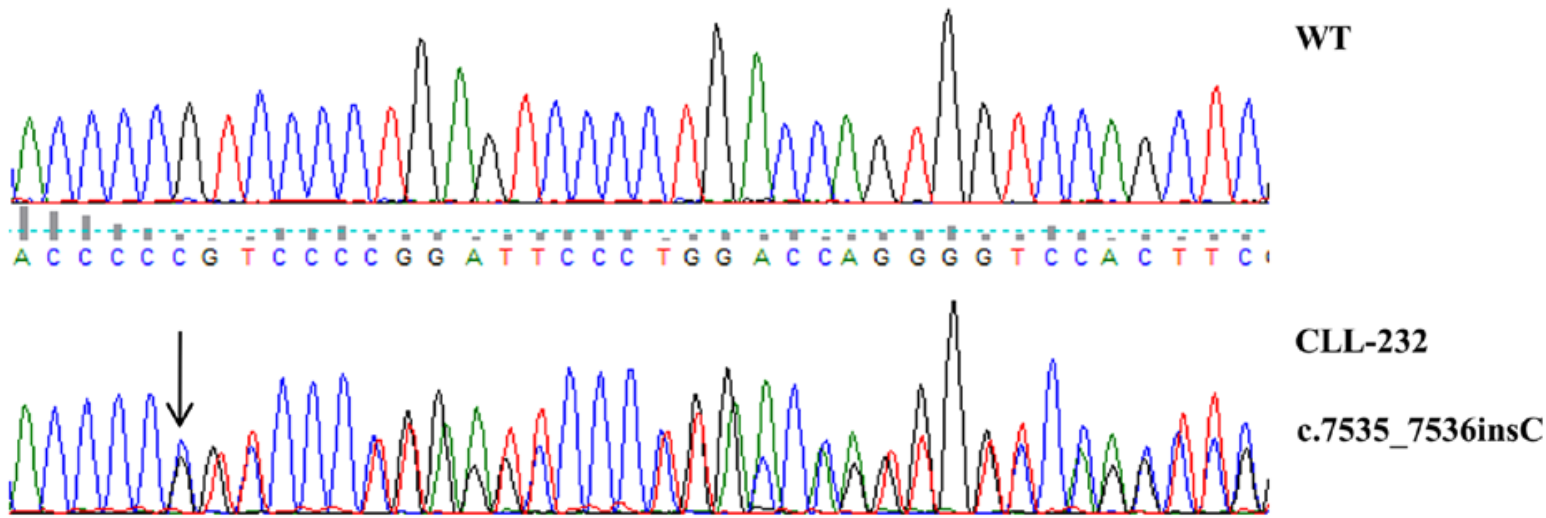

Figure 1. Melting curves and difference plots and sequencing traces for the PEST domain of NOTCH1. (A) The normalized and shifted melting curves of NOTCH1 showed that the HRM assay could distinguish the heterozygous genotype c.7541_7542delCT (CLL-108 in red) and heterozygous genotype c.7535_7536insC (CLL-232 in blue) from the wild-type. (B) The normalized and temp-shifted difference plots showed that the HRM could distinguish the heterozygous genotype c.7541_7542delCT (CLL-108 in red) and heterozygous genotype c.7535_7536insC (CLL-232 in blue) from the wild-type. Sequencing chromatograms confirmed (C) the heterozygous genotype c.7541_7542delCT in CLL-108) and (D) the heterozygous genotype c.7535_7536insC in CLL-232. HRM, high-resolution melting; CLL, chronic lymphocytic leukemia; WT wild-type.

mutation was detectable at a low peak by direct sequencing when the mutant frequency was $>10 \%$. However, when the mutation frequency was at $5 \%$, it was only distinguishable from the background. When the mutant frequency was $<5 \%$, the mutation was not detectable (Fig. 2A). Fig. 2B shows a normalized and shifted melting curves plot and normalized and temp-shifted difference plot of the HRM data. The melting curve from $1 \%$ mutant template sufficiently differed 


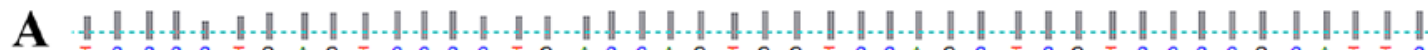
$T C C C C T G A G T C C C C T G A C C A G T G G T C C A G C T C G T C C C C G C A T T C$

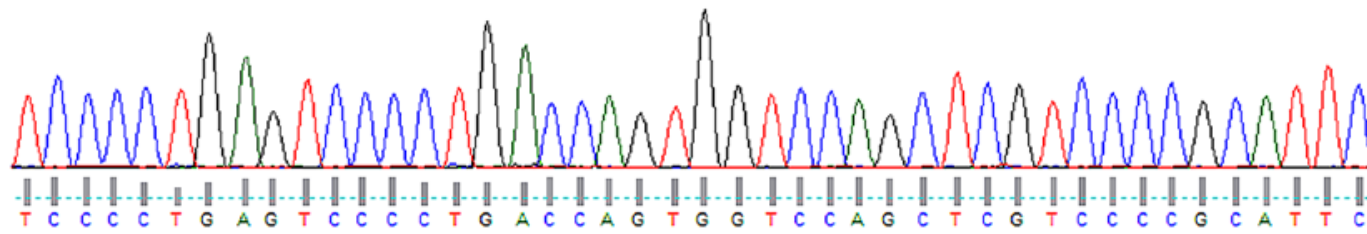

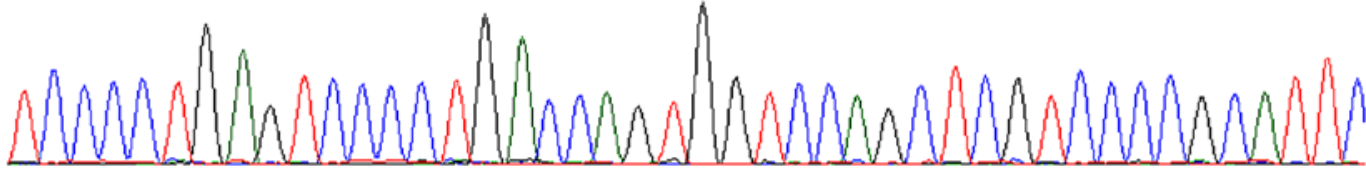

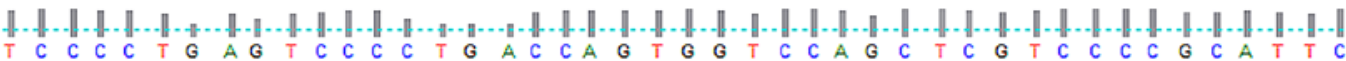

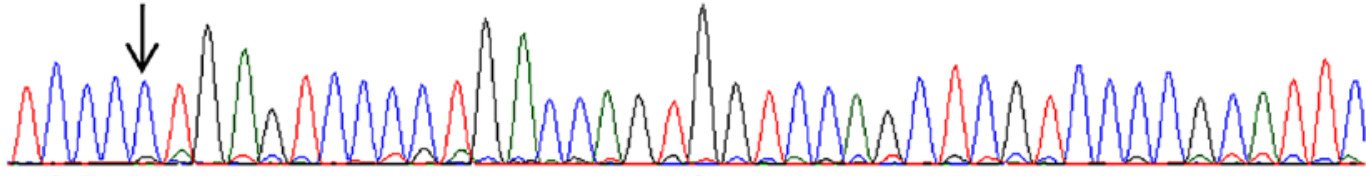

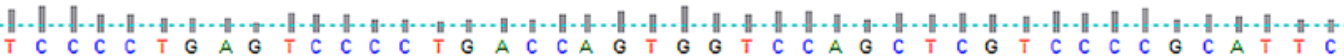

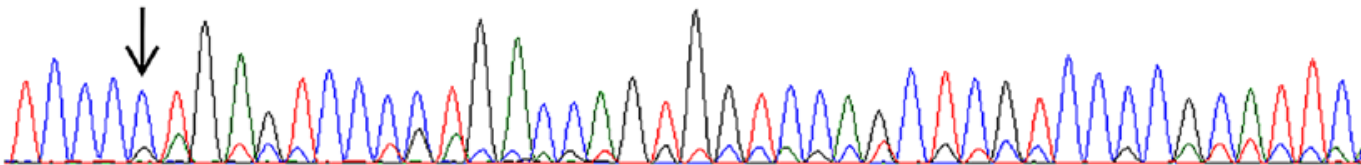

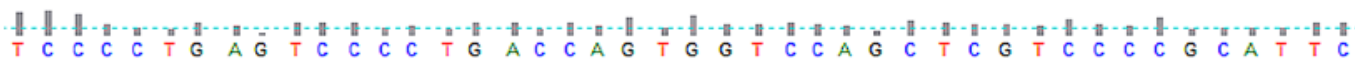

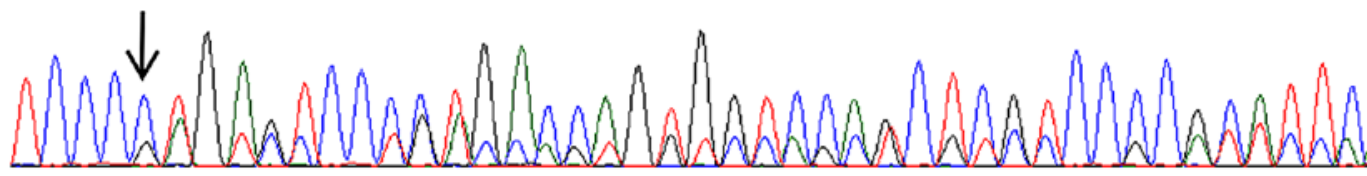

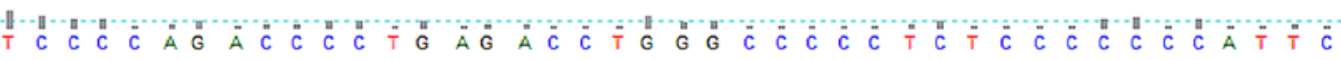
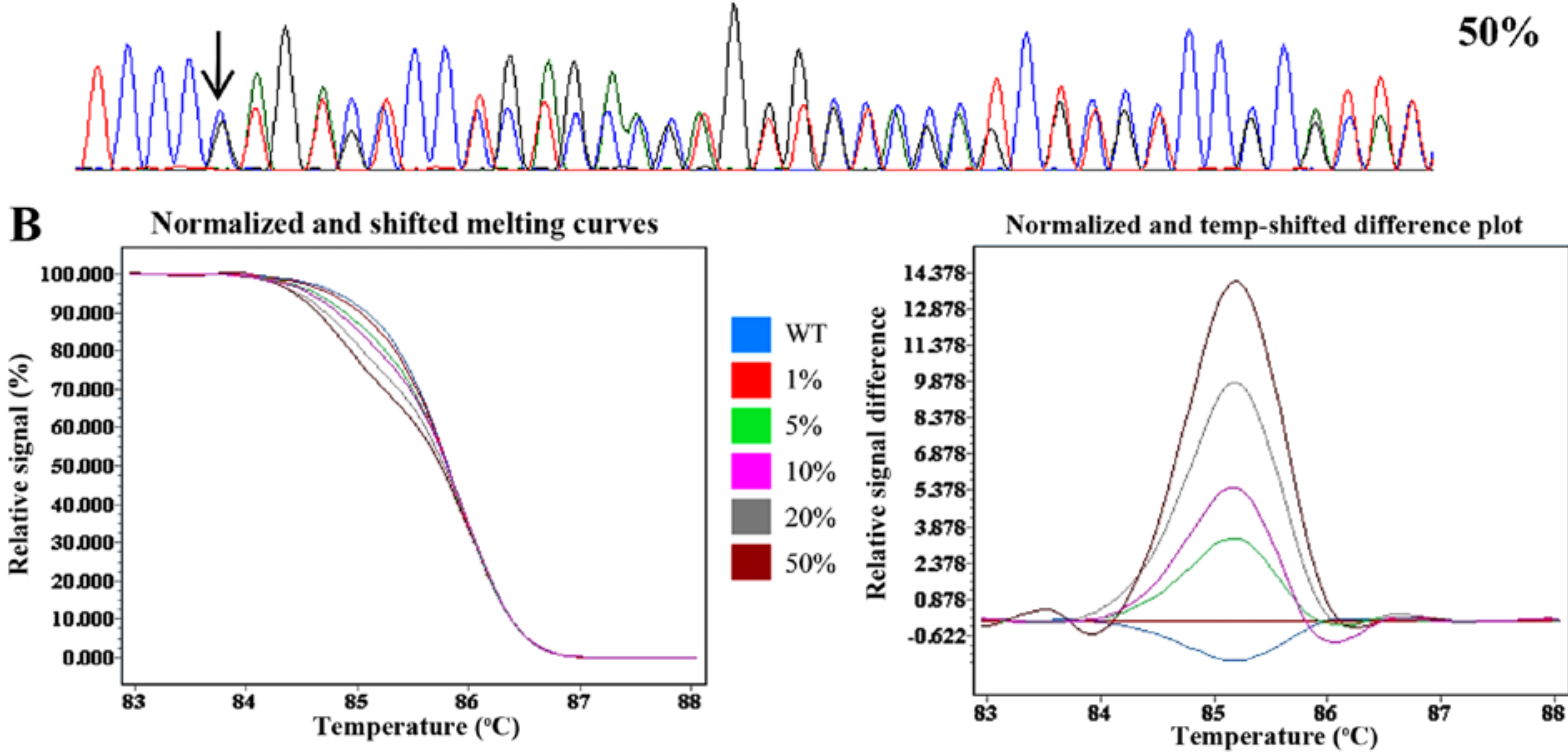

Figure 2. Validation and sensitivity testing for the HRM assay and direct sequencing. (A) Direct sequencing. At least $10 \%$ mutant gDNA was necessary to detect NOTCH1 mutations. (B) HRM. One percent mutant gDNA was necessary to be plotted differently from wild-type gDNA. HRM, high-resolution melting; gDNA, genomic DNA; WT, wild-type.

from the wild-type template, and this distinct melting profile was consistently observed across all other templates measured
$(5,10,20$ and $50 \%)$. Thus, the sensitivity of direct sequencing and HRM was found to be 10 and $1 \%$, respectively. 
Table IV. Comparison of direct sequencing and HRM assay.

\begin{tabular}{llcc}
\hline Methods & \multicolumn{1}{c}{ Facts } & $\begin{array}{c}\text { Sensitivity } \\
(\%)\end{array}$ & $\begin{array}{c}\text { Mutation analysis } \\
\text { methods TAT, } \mathrm{d}^{\mathrm{a}}\end{array}$ \\
\hline HRM & $\begin{array}{l}\text { Detects both known and unknown mutations; } \\
\text { inexpensive; requires sequencing validation } \\
\text { Detects every nucleotide change; expensive; } \\
\text { criterion standard }\end{array}$ & 1 & 0.5 \\
\hline
\end{tabular}

${ }^{a}$ The NOTCHI mutation analysis method TAT is defined as the time spent from measuring DNA extraction to the time that the results are generated from the instrument; it excludes the time spent for DNA extraction. TAT, turn-around time (d, days); HRM, high-resolution melting.

\section{Discussion}

The presence of NOTCH1 mutations disrupting the carboxyterminal PEST domain appears to confer adverse prognosis in CLL $(11,12,14,15)$. Evaluating the NOTCH1 mutation status is useful in clinical practice for patients with CLL and may facilitate therapeutic decision-making $(16,32,33)$. Therefore, selection of method for detecting somatic mutation with high sensitivity and specificity are of great importance. In this study, we successfully developed a powerful, highly sensitive, cost-effective and easy to perform approach for NOTCHI mutation screening in CLL. Most important, this is the first study to report NOTCH1 mutation detection in bone marrow samples of Chinese CLL patients using the HRM assay.

In the 133 newly diagnosed CLL patients, the melting curves of $6.02 \%$ (8/133) of the CLL patients were markedly different compared with the other 125 patients. The melting curves of 7 out of $8 \mathrm{NOTCH1}$-mutated patients were exactly the same. The 7 patients were confirmed to be c.7541_7542delCT mutated. The melting curve of 1 out of 8 NOTCH1-mutated patients was different. This patient was confirmed to be c.7535_7536insC mutated. All of the 133 CLL samples lacked single nucleotide polymorphisms (SNPs) according to the direct sequencing. SNPs are the result of genomic variation or changes of a single nucleotide in the genomic DNA. The HRM method has been successfully used in SNP genotyping and mutation detection $(34,35)$. Indeed, there are certain SNPs in the region of our PCR amplicons according to the dbSNP database. Unexpected SNPs may generated unique amplicon melting patterns $(36,37)$ (shifted melting curves and difference plot patterns) and be differentiated from either the c.7541_7542delCT or c.7535_7536insC mutation. Altogether, the HRM analysis used by us generated specific melting profiles that allowed the discrimination between wild-type and mutated samples (Fig. 1). Our HRM assay was confirmed to be reliable since all mutations detected by the HRM assay were also confirmed by direct sequencing.

In the present study, direct sequencing confirmed that $6.02 \%$ of the 133 Chinese CLL cases at diagnosis harbored NOTCH1 mutations in the PEST domain, and there were two genotypic heterozygous variations, c.7541_7542delCT (p.P2514fs"4) and c.7535_7536insC (p.S2513fs"3). The former mutation type comprised up to $87.5 \%$ (7/8) of the mutations. This frequency is consistent with a report in the Chinese population by Xia et al (38), but lower than that found in a series of studies of European CLL patients $(13,14,39)$. Therefore, there are discrepancies between Asian and European populations. CLL patients with mutated NOTCH1 showed a higher frequency of unmutated $I G H V$ and trisomy 12 , with statistically significant differences. This is in concordance with several previous studies (40-42).

The high sensitivity of the method was also confirmed in our experiments. Using cell lines with wild-type and heterozygous c.7541_7542delCT NOTCH1 mutation, we found that the sensitivity of HRM in our experimental setting was $1 \%$, while direct sequencing analysis had a sensitivity of $10 \%$. However, the frequency of the NOTCHI mutation detected by HRM was the same as that of direct sequencing, indicating that the mutational burden in our CLL cohort was $>10 \%$. Actually, the samples we analyzed were CLL monoclonal cells. Only CLL samples containing $\geq 95 \%$ of $\mathrm{CD}^{+} \mathrm{CD} 19^{+}$cells were included in the study.

Direct sequencing, known as the 'gold standard', has been used to detect somatic mutations for many years. It is able to detect any mutation in the DNA sequence being analyzed. But its limited sensitivity, high cost and long TAT have prompted the development of alternative methods for routine clinical testing which have greater diagnostic practicality for somatic mutation detection.

HRM is more sensitive, faster, less expensive and time saving than direct sequencing. In this study, the entire procedure needs $\sim 50 \mathrm{ng}$ gDNA and requires 0.5 days for the TAT, one sixth of the time compared to direct sequencing (Table IV). Another major advantage for HRM over direct sequencing is that it is performed in a 'closed tube' system. This eliminates the risk of post-PCR product contamination during scanning while also reducing processing time, since our PCR and HRM assay were performed within one instrument in the present study.

However, it has also been reported that this method can produce false-positive results due to bad DNA quality, particularly when the starting material is formalin-fixed, paraffin-embedded (FFPE) tissue (43). In the present study, the gDNA we detected was extracted from the bone marrow of the CLL patients, therefore the DNA quality was controllable. The concordance rate between HRM and sequencing was $100 \%$. There was only a slight false-positive chance for our analysis.

To the best of our knowledge, HRM analysis has already been applied for the diagnosis of tumors and genetic diseases. HRM analysis has been confirmed to a reliable, accurate, and rapid screening method for APC mutations in oral squamous cell carcinoma (44). BRCA1 gene screening using HRM 
analysis has been demonstrated to be useful for the diagnosis of lung adenocarcinoma (45) and Moroccan breast cancer (46); Studies using HRM analysis showed heterozygous mutations in COL1A1 and COL1A2 genes are associated with osteogenesis imperfecta (47).

In conclusion, our HRM assay opens a new avenue for the detection of NOTCHI gene mutations in CLL. It is a valid and promising tool for high-throughput NOTCH1 screening, enabling real-time evaluation of CLL progression, which is significant for decision-making regarding treatment.

\section{Acknowledgements}

The present study was supported by the National Natural Science Foundation of China (no. 81400154), the Natural Science Foundation of Jiangsu Province (no. BK20151211), and the Ninth Science and Technology Development Program of Suzhou (no. SYS201337).

\section{References}

1. Zenz T, Mertens D, Küppers R, Döhner H and Stilgenbauer S: From pathogenesis to treatment of chronic lymphocytic leukaemia. Nat Rev Cancer 10: 37-50, 2010.

2. Gaidano G, Foà R and Dalla-Favera R: Molecular pathogenesis of chronic lymphocytic leukemia. J Clin Invest 122: 3432-3438, 2012.

3. Chiorazzi N, Rai KR and Ferrarini M: Chronic lymphocytic leukemia. N Engl J Med 352: 804-815, 2005.

4. Tam CS and Keating MJ: Chemoimmunotherapy of chronic lymphocytic leukemia. Nat Rev Clin Oncol 7: 521-532, 2010.

5. Puente XS, Pinyol M, Quesada V, Conde L, Ordóñez GR, Villamor N, Escaramis G, Jares P, Beà S, González-Díaz M, et al: Whole-genome sequencing identifies recurrent mutations in chronic lymphocytic leukaemia. Nature 475: 101-105, 2011.

6. Rossi D, Fangazio M, Rasi S, Vaisitti T, Monti S, Cresta S, Chiaretti S, Del Giudice I, Fabbri G, Bruscaggin A, et al: Disruption of BIRC3 associates with fludarabine chemorefractoriness in TP53 wild-type chronic lymphocytic leukemia. Blood 119: 2854-2862, 2012.

7. Blaumueller CM, Qi H, Zagouras P and Artavanis-Tsakonas S: Intracellular cleavage of Notch leads to a heterodimeric receptor on the plasma membrane. Cell 90: 281-291, 1997.

8. Yuan JS, Kousis PC, Suliman S, Visan I and Guidos CJ: Functions of notch signaling in the immune system: Consensus and controversies. Annu Rev Immunol 28: 343-365, 2010.

9. Lobry C, Oh P and Aifantis I: Oncogenic and tumor suppressor functions of Notch in cancer: It's NOTCH what you think. J Exp Med 208: 1931-1935, 2011.

10. Lobry C, Oh P, Mansour MR, Look AT and Aifantis I: Notch signaling: Switching an oncogene to a tumor suppressor. Blood 123: 2451-2459, 2014.

11. Rosati E, Sabatini R, Rampino G, Tabilio A, Di Ianni M, Fettucciari K, Bartoli A, Coaccioli S, Screpanti I and Marconi P: Constitutively activated Notch signaling is involved in survival and apoptosis resistance of B-CLL cells. Blood 113: 856-865, 2009.

12. Arruga F, Gizdic B, Serra S, Vaisitti T, Ciardullo C, Coscia M, Laurenti L, D'Arena G, Jaksic O, Inghirami G, et al: Functional impact of NOTCH1 mutations in chronic lymphocytic leukemia. Leukemia 28: 1060-1070, 2014.

13. Fabbri G, Rasi S, Rossi D, Trifonov V, Khiabanian H, Ma J, Grunn A, Fangazio M, Capello D, Monti S, et al: Analysis of the chronic lymphocytic leukemia coding genome: Role of NOTCH1 mutational activation. J Exp Med 208: 1389-1401, 2011.

14. Rossi D, Rasi S, Fabbri G, Spina V, Fangazio M, Forconi F, Marasca R, Laurenti L, Bruscaggin A, Cerri M, et al: Mutations of NOTCH1 are an independent predictor of survival in chronic lymphocytic leukemia. Blood 119: 521-529, 2012.

15. Weissmann S, Roller A, Jeromin S, Hernández M, Abáigar M, Hernández-Rivas JM, Grossmann V, Haferlach C, Kern W, Haferlach T, et al: Prognostic impact and landscape of NOTCH1 mutations in chronic lymphocytic leukemia (CLL): A study on 852 patients. Leukemia 27: 2393-2396, 2013.
16. López-Guerra M, Xargay-Torrent S, Rosich L, Montraveta A, Roldán J, Matas-Céspedes A, Villamor N, Aymerich M, López-Otín C, Pérez-Galán P, et al: The $\gamma$-secretase inhibitor PF-03084014 combined with fludarabine antagonizes migration, invasion and angiogenesis in NOTCH1-mutated CLL cells. Leukemia 29: 96-106, 2015.

17. Osipo C, Golde TE, Osborne BA and Miele LA: Off the beaten pathway: The complex cross talk between Notch and NF-kappaB. Lab Invest : 11-17, 2008.

18. Di Ianni M, Baldoni S, Rosati E, Ciurnelli R, Cavalli L, Martelli MF, Marconi P, Screpanti I and Falzetti F: A new genetic lesion in B-CLL: A NOTCH1 PEST domain mutation. Br J Haematol 146: 689-691, 2009.

19. Sportoletti P, Baldoni S, Cavalli L, Del Papa B, Bonifacio E, Ciurnelli R, Bell AS, Di Tommaso A, Rosati E, Crescenzi B, et al: NOTCH1 PEST domain mutation is an adverse prognostic factor in B-CLL. Br J Haematol 151: 404-406, 2010.

20. Sportoletti P, Baldoni S, Del Papa B, Aureli P, Dorillo E, Ruggeri L, Plebani S, Amico V, Di Tommaso A, Rosati E, et al: A revised NOTCH1 mutation frequency still impacts survival while the allele burden predicts early progression in chronic lymphocytic leukemia. Leukemia 28: 436-439, 2014.

21. Stilgenbauer S, Schnaiter A, Paschka P, Zenz T, Rossi M, Döhner K, Bühler A, Böttcher S, Ritgen M, Kneba M, et al: Gene mutations and treatment outcome in chronic lymphocytic leukemia: Results from the CLL8 trial. Blood 123: 3247-3254, 2014.

22. Oscier DG, Rose-Zerilli MJ, Winkelmann N, Gonzalez de Castro D, Gomez B, Forster J, Parker H, Parker A, Gardiner A, Collins A, et al: The clinical significance of NOTCH1 and SF3B1 mutations in the UK LRF CLL4 trial. Blood 121: 468-475, 2013.

23. Puente XS, Beà S, Valdés-Mas R, Villamor N, Gutiérrez-Abril J, Martín-Subero JI, Munar M, Rubio-Pérez C, Jares P, Aymerich M, et al: Non-coding recurrent mutations in chronic lymphocytic leukaemia. Nature 526: 519-524, 2015.

24. Gundry CN, Vandersteen JG, Reed GH, Pryor RJ, Chen J and Wittwer CT: Amplicon melting analysis with labeled primers: A closed-tube method for differentiating homozygotes and heterozygotes. Clin Chem 49: 396-406, 2003.

25. Reed GH, Kent JO and Wittwer CT: High-resolution DNA melting analysis for simple and efficient molecular diagnostics. Pharmacogenomics 8: 597-608, 2007.

26. Taylor CF: Mutation scanning using high-resolution melting. Biochem Soc Trans 37: 433-437, 2009.

27. Liew M, Pryor R, Palais R, Meadows C, Erali M, Lyon E and Wittwer C: Genotyping of single-nucleotide polymorphisms by high-resolution melting of small amplicons. Clin Chem 50: 1156-1164, 2004

28. Reed GH and Wittwer CT: Sensitivity and specificity of singlenucleotide polymorphism scanning by high-resolution melting analysis. Clin Chem 50: 1748-1754, 2004.

29. Graham R, Liew M, Meadows C, Lyon E and Wittwer CT: Distinguishing different DNA heterozygotes by high-resolution melting. Clin Chem 51: 1295-1298, 2005.

30. Herrmann MG, Durtschi JD, Bromley LK, Wittwer CT and Voelkerding KV: Amplicon DNA melting analysis for mutation scanning and genotyping: Cross-platform comparison of instruments and dyes. Clin Chem 52: 494-503, 2006.

31. Montgomery J, Wittwer CT, Palais R and Zhou L: Simultaneous mutation scanning and genotyping by high-resolution DNA melting analysis. Nat Protoc 2: 59-66, 2007.

32. Wei P, Walls M, Qiu M, Ding R, Denlinger RH, Wong A, Tsaparikos K, Jani JP, Hosea N, Sands M, et al: Evaluation of selective gamma-secretase inhibitor PF-03084014 for its antitumor efficacy and gastrointestinal safety to guide optimal clinical trial design. Mol Cancer Ther 9: 1618-1628, 2010.

33. Wu Y, Cain-Hom C, Choy L, Hagenbeek TJ, de Leon GP, Chen Y, Finkle D, Venook R, Wu X, Ridgway J, et al: Therapeutic antibody targeting of individual Notch receptors. Nature 464: 1052-1057, 2010.

34. Schmidt U, Hulkkonen J and Naue J: Detection of a $\mathrm{G}>\mathrm{C}$ single nucleotide polymorphism within a repetitive DNA sequence by high-resolution DNA melting. Int J Legal Med: Mar 14, 2016 (Epub ahead of print).

35. Hong Y, Pandey MK, Liu Y, Chen X, Liu H, Varshney RK, Liang $X$ and Huang S: Identification and evaluation of singlenucleotide polymorphisms in allotetraploid peanut (Arachis hypogaea $\mathrm{L}$.) based on amplicon sequencing combined with high resolution melting (HRM) analysis. Front Plant Sci 6: 1068, 2015. 
36. de Juan I, Esteban E, Palanca S, Barragán E and Bolufer P. High-resolution melting analysis for rapid screening of BRCA1 and BRCA2 Spanish mutations. Breast Cancer Res Treat 115: 405-414, 2009.

37. Er TK and Chang JG: High-resolution melting: Applications in genetic disorders. Clin Chim Acta 414: 197-201, 2012.

38. Xia Y, Fan L, Wang L, Gale RP, Wang M, Tian T, Wu W, Yu L, Chen YY, Xu W, et al: Frequencies of SF3B1, NOTCH1, MYD88, BIRC3 and IGHV mutations and TP53 disruptions in Chinese with chronic lymphocytic leukemia: Disparities with Europeans. Oncotarget 6: 5426-5434, 2015.

39. Shedden K, Li Y, Ouillette P and Malek SN: Characteristics of chronic lymphocytic leukemia with somatically acquired mutations in NOTCH1 exon 34. Leukemia 26: 1108-1110, 2012.

40. Balatti V, Bottoni A, Palamarchuk A, Alder H, Rassenti LZ, Kipps TJ, Pekarsky Y and Croce CM: NOTCH1 mutations in CLL associated with trisomy 12. Blood 119: 329-331, 2012.

41. Del Giudice I, Rossi D, Chiaretti S, Marinelli M, Tavolaro S, Gabrielli S, Laurenti L, Marasca R, Rasi S, Fangazio M, et al: NOTCH1 mutations in +12 chronic lymphocytic leukemia (CLL) confer an unfavorable prognosis, induce a distinctive transcriptional profiling and refine the intermediate prognosis of +12 CLL. Haematologica 97: 437-441, 2012.

42. Villamor N, Conde L, Martínez-Trillos A, Cazorla M, Navarro A, Beà $\mathrm{S}$, López $\mathrm{C}$, Colomer $\mathrm{D}$, Pinyol $\mathrm{M}$, Aymerich $\mathrm{M}$, et al: NOTCH1 mutations identify a genetic subgroup of chronic lymphocytic leukemia patients with high risk of transformation and poor outcome. Leukemia 27: 1100-1106, 2013.
43. Do H, Krypuy M, Mitchell PL, Fox SB and Dobrovic A: High resolution melting analysis for rapid and sensitive EGFR and KRAS mutation detection in formalin fixed paraffin embedded biopsies. BMC Cancer 8: 142, 2008.

44. Marino M, Monzani ML, Brigante G, Cioni K, Madeo B, Santi D, Maiorana A, Bettelli S, Moriondo V, Pignatti E, et al: High-resolution melting is a sensitive, cost-effective, time-saving technique for BRAF V600E detection in thyroid FNAB washing liquid: A prospective cohort study. Eur Thyroid J 4: 73-81, 2015.

45. Wright GM, Do H, Weiss J, Alam NZ, Rathi V, Walkiewicz M, John T, Russell PA and Dobrovic A: Mapping of actionable mutations to histological subtype domains in lung adenocarcinoma: Implications for precision medicine. Oncotarget 5: 2107-2115, 2014

46. El Khachibi M, Diakite B, Hamzi K, Badou A, Senhaji MA, Bakhchane A, Jouhadi H, Barakat A, Benider A and Nadifi S: Screening of exon 11 of BRCA1 gene using the high resolution melting approach for diagnosis in Moroccan breast cancer patients. BMC Cancer 15: 81, 2015.

47. Wang J, Ren X, Bai X, Zhang T, Wang Y, Li K and Li G: Identification of gene mutation in patients with osteogenesis imperfecta using high resolution melting analysis. Sci Rep 5: 13468, 2015. 